\title{
L'epatite E in dialisi
}

\author{
F. Fabrizi ${ }^{1}$, G. Lunghi ${ }^{2}$, F. Locatelli ${ }^{3}$ \\ ${ }^{1}$ Divisione di Nefrologia e Dialisi, ${ }^{2}$ Istituto di Igiene e Preventiva, Ospedale Maggiore \\ Policlinico, IRCCS, Milano, ${ }^{3}$ Divisione di Nefrologia e Dialisi, Ospedale di Lecco, Lecco
}

infezione da virus dell'epatite $\mathrm{E}(\mathrm{HEV})$ causa una epatite acuta che in precedenza era denominata epatite non-A, non-B a trasmissione enterale. Si tratta di un'epatite itterica che di norma non produce sequele ma può essere severa, colpisce generalmente giovani adulti ed adolescenti, e ha un tasso di mortalità piuttosto elevato (fino al $20 \%$ ) nelle giovani donne che contraggono l'infezione durante il terzo trimestre di gravidanza.

Una discreta mole di dati riguardo l'epidemiologia di HEV nell'uomo è stata prodotta nel corso degli anni '90, dopo l'isolamento del virus (1) e la messa a punto di metodiche diagnostiche (2). La via fecale-orale rappresenta la più importante modalità di trasmissione di HEV; è stata però evidenziata una fase di transitoria viremia in volontari sani che dopo avere ingerito il virus avevano sviluppato epatite E (3). Tale osservazione ha indotto a ipotizzare una via parenterale di trasmissione di HEV, accanto alla trasmissione fecale-orale; sono state pertanto condotte ricerche riguardo l'epidemiologia di HEV nei pazienti in dialisi di mantenimento (4-13), visto che tale popolazione è ad alto rischio di acquisire infezioni a via parenterale.

\section{Morfologia di HEV}

Il virus dell'epatite $\mathrm{E}$ presenta dimensioni, forma e caratteristiche fisicochimiche simili ai calicivirus nei quali è stato classificato (14). È stato di recente dimostrato come HEV abbia una considerevole eterogeneità genetica e ciò ha indotto alla classificazione dei vari genotipi HEV. I più importanti genotipi sono i seguenti: genotipo 1 (Burmese), genotipo 2 (Messicano), genotipo 3 (Nord America), genotipo 4 (Cina e Taiwan), genotipo 5 (identificato in Italia), genotipi 6 e 7 (isolati in Grecia) (15). Non è stato ancora allestito un sistema immunoenzimatico in grado di identificare tutti i genotipi isolati. Non sembra esservi relazione tra virulenza e genotipi HEV. HEV sembra avere stabilità in vivo, al contrario di quanto è stato osservato in vitro.

Non esistono sistemi di coltura cellulare per $\mathrm{HEV}$; al fine di studiare patogenesi e progressione della malattia śono stati usati vari modelli animali quali: scimpanzè (Pan Troglodytes), tamarindo (Sanguinus Mystax) e scimmie (Macaca Mulata).

\section{Distribuzione geografica}

Epidemie di HEV sono state osservate in India, Pakistan, Nepal e anche nell'Asia centrale e Africa dell'est. Focolai epidemici più limitati sono stati riportati in molte regioni tropicali. Casi sporadici di epatite E sono stati osservati in regioni industrializzate quali Regno Unito, USA, Germania, Olanda e Giappone; tali casi sono di solito correlati a una anamnesi positiva per recenti viaggi in aree di endemia per HEV. Non vi sono differenze nella presentazione clinica di casi di epatite $E$ nei paesi industrializzati rispetto a quelli in via di sviluppo. In Italia esiste una circolazione piuttosto bassa di HEV: la prevalenza di anticorpi anti-HEV (segno di contagio con il virus HEV) è stata pari allo $0.95 \%$ tra i donatori di sangue, $0.74 \%$ nella popolazione generale e $1.94 \%$ tra i tossicodipendenti (16). La frequenza di anticorpi anti-HEV sembra essere più alta nelle regioni meridionali del nostro paese; nella popolazione generale della zona di Napoli è stata osservata una prevalenza di anticorpi anti-HEV pari al $14 \%$ (17). La prevalenza media di anticorpi anti-HEV nei donatori di sangue Europei è dell' $1.3 \%$. 


\section{Epidemiologia di $\mathrm{HEV}$}

L'infezione da HEV si trasmette per via fecale-orale principalmente attraverso acqua potabile contaminata; meno frequenti e più circoscritte sono risultate le epidemie dovute a cibo inquinato dal virus. La trasmissione del virus da persona a persona è stata evidenziata durante le epidemie di HEV ma sembra accadere con una frequenza ridotta rispetto ad altre infezioni enteriche virali quali, ad esempio l'epatite A. È stata anche osservata la trasmissione di HEV tramite trasfusioni, per via sessuale o placentare. Da alcuni autori l'abuso di stupefacenti per via intravenosa e i trattamenti dentari sono stati implicati come fattori di rischio in zone non a rischio di endemia per HEV.

\section{Diagnosi di infezione da HEV}

L'esistenza di infezione da HEV è stata sospettata nel corso degli anni Ottanta quando sono state descritte epidemie di epatite acuta a trasmissione fecale-orale che non erano dovute ai virus dell'epatite A o B (1). Il virus HEV è stato all'inizio identificato mediante microscopia elettronica. Nel corso della prima metà degli anni Novanta sono stati allestiti test immunoenzimatici (2) per la diagnosi di HEV in grado di accertare la presenza nel siero di anticorpi rivolti contro antigeni ricombinanti o peptidi sintetici del virus (anticorpi anti-HEV). Risultati ripetutamente positivi ottenuti con i test immunoenzimatici sono di solito confermati con test supplementari che offrono maggiore specificità, basati su una metodica western blot (immunoblot) che utilizza antigeni ricombinanti di HEV. La presenza di anticorpi nel siero anti-HEV è espressione di contatto con il virus. La ricognizione diretta del virus nel sangue può essere compiuta mediante reazione a catena dell'enzima polimerasi accompagnata da retrotrascrizione inversa (RT-PCR).

\section{HEV nei pazienti in dialisi periodica}

Non sono stati fino ad ora descritti focolai epidemici di epatite E nei pazienti sottoposti ad emodialisi di mantenimento. Gli studi riguardo l'epidemiologia dell'infezione da HEV nei pazienti in emodialisi periodica sono di numero ristretto e su piccoli gruppi di pazienti $(4,13)$. Complessivamente è stato studiato un totale di 1660 pazienti, principalmente delle regioni industrializzate, con una frequenza di anticorpi anti-HEV tra $0 \%$ e $17 \%$. Varie cause possono spiegare tale ampia variabilità di risultati: le caratteristiche demografiche della popolazione studiata, la numerosità del campione analizzato, la prevalenza di HEV nella popolazione generale corrispondente, i criteri di inclusione dei pazienti oggetto di studio ed altri fattori non ancora chiariti. In uno studio preliminare (4) è stata riportata una prevalenza piuttosto alta di anticorpi anti-HEV (10.8\%) ed è stato evidenziato il concetto che la via fecale-orale non sia la via esclusiva di trasmissione di HEV nei dializzati: questi autori ipotizzarono una diffusione di HEV simile a quella di altri virus epatotropi quali $\mathrm{HBV}$ e HCV. Tuttavia, studi successivi non hanno confermato un'alta prevalenza di anticorpi anti-HEV $(5,6,10,12)$; oppure non è stata osservata differenza nella frequenza di anticorpi antiHEV tra dializzati e gruppo di controllo (13). In altre indagini è stata osservata un'aumentata frequenza di anticorpi anti-HEV negli emodializzati rispetto alla popolazione generale ma ciò era dovuto all'effetto confondente di età e sesso (8); oppure mancava il gruppo di controllo (9) o una dettagliata informazione demografica (12). Ancora, un'elevata prevalenza di infezione HEV potrebbe essere il risultato di focolai infettivi locali.

Non è stata trovata associazione tra anticorpi anti-HEV e caratteristiche demografiche, cliniche o biochimiche dei pazienti quali: età anagrafica, sesso, razza, tempo di dialisi, anamnesi trasfusionale, alterazioni degli enzimi epatici, storia clinica di epatite e positività per markers $\mathrm{HBV} / \mathrm{HCV}$.

Uno studio ha valutato l'incidenza di HEV in un gruppo di pazienti sottoposti ad emodialisi periodica: nessun caso di sieroconversione per HEV è stato identificato (11). Non sono state pubblicate indagini circa l'epidemiologia di HEV nei pazienti sottoposti a dialisi peritoneale, nei soggetti con trapianto renale funzionante oppure negli individui affetti da insufficienza

\section{TABLE I - PREVALENZA DI ANTICORPI ANTI-HEV NEI PAZIENTI IN DIALISI PERIODICA}

Autore

Frequenza anti-HEV

(\%)

Halfon P, et al.

$16 / 147$

10.9

Courtney MG, et al.

$0 / 45$

0

Macedo G, et al.

$3 / 68$

4.4

Buti MG, et al.

$3 / 50$

Psichigiou M, et al.

$27 / 420$

6

Gessoni G, et al.

$18 / 193$

6.4

Fabrizi F, et al.

$6 / 204$

9.3

Sylvan SPE, et al.

11/182

Dalekos GN, et al.

$2 / 149$

Dalekos GN, et al.

$6 / 62$

Arinsoy $\mathrm{T}$, et al.

$9 / 52$

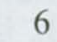

1.3

9.7

17.3 
renale cronica in fase conservativa. Tutte le ricerche circa HEV nei pazienti in dialisi sono state compiute mediante test sierologici; non sono state svolte sino ad ora ricerche con PCR. Viaggiare non è comune tra $\mathrm{i}$ pazienti in emodialisi a causa della necessità di sottoporsi alla terapia tre volte alla settimana; questo potrebbe contribuire a spiegare la bassa frequenza di infezione nei dializzati delle regioni industrializzate.

\section{Conclusioni}

Le indagini riguardo l'epidemiologia di HEV nei pazienti in dialisi di mantenimento sono piuttosto limitate. L'informazione disponibile non supporta la nozione di un ruolo definito di HEV nella patogenesi dell'epatopatia in questa popolazione. Molte domande rimangono irrisolte: l'epidemiologia di HEV negli emodializzati che vivono in regioni endemiche per HEV, l'impatto clinico dei genotipi HEV nei dializzati infetti, la persistenza lungo il tempo degli anticorpi anti-HEV (11), la possibile presenza di viremia HEV in pazienti anti-HEV negativi.

$\mathrm{Al}$ momento l'epatite $\mathrm{E}$ non è un problema importante di salute pubblica nei paesi sviluppati; tuttavia il crescente numero di viaggiatori verso aree di endemia per HEV e l'alto flusso di immigranti da queste regioni richiede una sorveglianza attenta nella popolazione generale. Si raccomanda un periodico screening per gli anticorpi anti-HEV nei pazienti a rischio di sviluppare infezioni virali, quali i pazienti in emodialisi periodica.

\section{BIBLIOGRAFIA}

1. Reyes GR, Purdy MA, Kim JP, et al. Isolation of a cDNA from the virus responsible for enterically transmitted non-A, non-B hepatitis. Science 1990; 247: 1335-9.

2. Dawson GJ, Chau KH, Cabal CM, Yarbough PO, Reyes GR, Mushahwar IK. Solid phase enzymelinked immunosorbent assay for hepatitis E virus IgG and IgM antibodies utilizing recombinant antigens and synthetic peptides. J Virol Methods 1992; 38: 175-86.

3. Chauhan A, Jameel S, Dilawari GB, Chawla YK, Kaur U, Ganguly NK. Hepatitis E virus transmission to a volunteer. Lancet 1993; 341: 149-50.

4. Halfon P, Ouzan D, Chanas M, et al. High prevalence of hepatitis $E$ virus antibody in hemodialysis patients. Lancet 1994; 344: 746 (letter).

5. Courtney MG, O'Mahoney M, Albloushi S, et al. Hepatitis E virus antibody prevalence in hemodialysis patients. Lancet 1994; 344: 1166 (letter).

6. Macedo G, Chaves AP, Pinto T, et al. Prevalence of hepatitis E virus antibody in haemodialysis patients. Nephrol Dial Transplant 1995; 10: 1017A.

7. Buti M, Jardi R, Cotrina M, et al. Hepatitis $E$ virus infection in acute hepatitis in Spain. J Virol Methods 1995; 55: 48-54.

8. Psichigiou M, Vaindirli E, Tzala E, et al. for the Multicentre Haemodialysis Cohort Study on Viral Hepatitis. Hepatitis E virus (HEV) infection in haemodialysis patients. Nephrol Dial Transplant 1996; 11 : 1093-5.

9. Gessoni G, Manoni F. Hepatitis $E$ virus infection in north-east Italy: serological study in the open population and groups at risk. $\mathrm{J}$ Hepatitis 1996; 11: 1093-5.

10. Fabrizi F, Lunghi G, Bacchini
G, Corti M, Pagano A, Locatelli F. Hepatitis $E$ virus infection in haemodialysis patients: a seroepidemiological survey. Nephrol Dial Transplant 1997; 12: 133-6.

11. Sylvan SPE, Jacobson SH, Christenson B. Prevalence of antibodies to hepatitis $\mathrm{E}$ virus among hemodialysis patients in Sweden. J Med Virol 1998; 54: 38-43.

12. Dalekos GN, Zervou E, Elisaf $\mathrm{M}$, et al. Antibodies to hepatitis E virus among several populations in Greece: increased prevalence in a hemodialysis unit. Transfusion 1998; 38: 589-95.

13. Arinsoy T, Yilmaz M, Derici U, Sindel S, Tekin I, Hasanoglu E. Prevalence of hepatitis E virus antibody in hemodialysis patients. Nephron 1998; 80: 85 (letter).

14. Bradley DW, Balayan MS. Virus of enterically transmitted non-A, non-B hepatitis. Lancet 1988; 1 : 819.

15. Fabrizi F, Martin P. Hepatitis E virus in end-stage renal disease. Int J Artif Organs 1999; 22: 593-9.

16. Zanetti AR, Dawson GJ, and the Study Group of Hepatitis E. Hepatitis type E in Italy: a seroepidemiological survey. J Med Virol 1994; 42: 318-20.

17. Pisanti FA, Coppola A, Galli C. Association between hepatitis $\mathrm{C}$ and hepatitis $E$ viruses in southern Italy. Lancet 1994; 344: 746-7.

18. Khuroo MS, Kamili S, Dar MY, Moecklii R, Jameel S. Hepatitis $\mathrm{E}$ and long-term antibody status. Lancet 1993; 341: 1355 (letter). 